\title{
Risky Donation for Rewarding Innovation? Examining Transformation of Technology Consumers into Crowdfunding Patrons
}

\author{
Shankhadeep Banerjee \\ Management Information Systems \\ Indian Institute of Management Calcutta \\ Kolkata, India \\ Email: shankhadeepb15@iimcal.ac.in

\section{Indranil Bose} \\ Management Information Systems \\ Indian Institute of Management Calcutta \\ Kolkata, India \\ Email: bose@iimcal.ac.in
}

\begin{abstract}
Reward-based crowdfunding platforms transform technophilic consumers into technology patrons by enabling them to donate for technology development in lieu of receiving the finished product as reward in future. Literature specifically on crowdfunding of technologies is tenuous, and researchers have not yet established the causal factors which entice technology consumers to donate. Using Elaboration Likelihood Model as theoretical base, we conduct a 2x2x2 mixed-design experiment to examine the effects of three core elements of crowdfunding (value of reward, waiting time to receive technology, and affective cues in donor appeal) on a potential consumer's likelihood to donate. Our results show positive impact of reward, and negative impact of waiting time, but surprisingly no effect of affective cues. Significant interaction between reward and waiting time has also been observed. Apart from theoretical contributions, the findings have tactical implications for technology start-ups planning to raise funding through donations, and design implications for crowdfunding platforms.
\end{abstract}

Keywords Reward-based crowdfunding, Donation, Technology Patron, Technology Funding, Elaboration Likelihood Model 


\section{Introduction}

Crowdfunding refers to "the efforts by entrepreneurial individuals and groups-cultural, social, and forprofit-to fund their ventures by drawing on relatively small contributions from a relatively large number of individuals using the internet, without standard financial intermediaries" (Mollick, 2014). Based on what the beneficiaries are willing to give to their backers in exchange of funding, and the amount of risk involved for the backers, crowdfunding can be classified into different models like private equity (e.g. EquityNet.com), royalty (e.g. Sellaband.com), microfinance (e.g. Kiva.com), peer-to-peer (e.g. LendingClub.com), rewards (e.g. Kickstarter.com), and donation (e.g. Experiment.com) (Beaulieu et al., 2015). In this paper, we focus on the reward-based crowdfunding model, where the backers get some non-monetary reward from the beneficiaries in exchange of their contribution. The reward can be small (thank you card, key chain, t-shirt, etc.) or big (one or more products and/or accessories) based on the amount of contribution. Reward-based crowdfunding platforms have grown in popularity over last years and they have pumped billions of dollars into thousands of innovative projects; e.g. from its conception to mid-2018, Kickstarter has successfully funded $145 \mathrm{~K}$ projects with $14 \mathrm{M}$ backers contributing US\$ 3.7B (Kickstarter, 2018). These projects can fall into several categories like Music, Film, Art, Food, Photography, Publishing, etc. and typically researchers even in the domain of Information Systems include all of them in studying crowdfunding phenomenon (Bretschneider and Leimeister, 2017; Kim et al., 2017; Zhao et al., 2018; Zheng et al., 2018). However, we choose to focus on the category of Technology, not just because it is directly relevant to IS but also because, interestingly, it has got the lowest success rate of raising money (just 20\% in Kickstarter compared to overall average of $36 \%$ ) among all categories and hence deserves special attention.

Reward-based crowdfunding of technologies is itself a multi-billion dollar phenomenon with US\$ 0.7B funding just in Kickstarter (Kickstarter, 2018), and yet research on it is scarce. It has been argued that the lack of a coherent research agenda, and standardized concepts have led to missing out on examining various core aspects of this phenomenon, and it has been proposed to start off with differentiating between backers who participate in crowdfunding for financial returns (technology investors) and those who receive non-financial or no returns (technology patrons) (Banerjee and Bose, 2017). In the literature relating to technology patrons, specific themes have been covered, e.g. effects of project quality signals and e-word-of-mouth on contribution decision (Bi et al., 2017), overfunding for technology projects (Cordova et al., 2015), impact of funding patterns on the performance of technology entrepreneurs (Jung et al., 2014), etc. However, most of the fundamental questions centred on the patron psychology and behaviour are yet to be investigated. For instance, what propels technology consumers to become technology patrons in the context of reward-based crowdfunding, especially when the reward itself is receiving the technology in future? Consumers can easily take the risk-free path of waiting for the targeted technologies to launch and then decide whether to purchase them or not. Is it because of intrinsic motivations like altruism, or extrinsic ones like rewards, or a mix of both? Also, do the core characteristics of reward-based crowdfunding process (like reward proposition) significantly trigger these motivations? The answers to these are important to the businesses interested in raising adequate funding from reward-based crowdfunding platforms. And so to investigate these, we frame our overarching research question as:

RQ1: How do certain core elements of reward-based crowdfunding impact a business' likelihood of receiving donation (for developing technology) from a potential consumer promised of being rewarded with the technology in future?

We chose three critical aspects of reward-based crowdfunding: proposed reward/discount (relative difference between expected retail price and requested donation), waiting time (temporal distance between the time of donation and the proposed delivery of technology), and affective cues in the donation request to evoke altruistic and technophilic emotions. Using Elaboration Likelihood Model, we expected the first two factors to affect a potential consumer's likelihood to donate through central route (high cognitive processing of information), while the last factor to affect the same via peripheral route (heuristics-based low cognitive processing). Since, in reality, these factors act simultaneously, there is a chance of significant interaction effects among them as well. Hence, we used a 2x2x2 mixedfactorial experiment with Latin Square design (Grant, 1948) to test the individual and collective impact of the three factors on the donation likelihood. We included adequate controls related to product, demographics, psychographics, and experiment design to ensure sufficient internal and external validity. We used a general linear mixed-effects model with random and repeated effects for subjects to analyse the data collected from a sample $(\mathrm{N}=114)$ of students and professionals (each presented with 4 within-subjects treatment scenarios, leading to 456 observations in total). Our results show positive impact of reward, and negative impact of waiting time, but surprisingly no effect of affective cues. Significant interaction between reward and waiting time has also been observed. 
This study makes several theoretical contributions: first, it extends the nascent but growing research on technology patrons and reward-based crowdfunding context; second, using experiment it establishes certain causal factors and their interactions that affect donation likelihood; third, it demonstrates an application of ELM theory in crowdfunding context; fourth, it introduces technology-based variables like technology attractiveness and technology complexity which can be used in innovation/technologycentric studies in various contexts. The findings have potential tactical implications for technology startups planning to raise funding through donations as they can tailor their crowdfunding request plans based on the significance of various factors. Similarly, the identification of certain critical factors for converting consumers to patrons also has design implications for crowdfunding platforms.

The upcoming sections would elaborate on the theoretical background, hypotheses development, experiment design, analyses, and results, and finally conclude with a discussion on findings, implications, limitations, and future research.

\section{Theory and Hypotheses}

Our research aims to understand how potential technology consumers process certain information presented to them in the context of reward-based crowdfunding and get persuaded to make donations. Hence, we chose a persuasion-related theory of Elaboration Likelihood Model (ELM) which explains different ways of people process information for decision-making (Petty and Cacioppo, 1983). Elaboration refers to "the extent to which a person scrutinizes the issue-relevant arguments contained in the persuasive communication" (Petty and Cacioppo, 1986). ELM proposes two routes of persuasion: the central route where "persuasion will likely result from a person's careful and thoughtful consideration of the true merits of the information presented in support of an advocacy"; and the peripheral route where "persuasion results from a person's association with positive or negative cues in the stimulus" (Petty and Cacioppo, 1984). Thus, the central route involves much more cognitive efforts (elaborations) as compared to the peripheral route. Factors in both these routes can not only directly affect decision making and attitudinal change, but even some of the interactions among those factors have also been found to be significant in various studies (Kim and Benbasat, 2009; Ma et al., 2013). ELM has been mostly used in marketing domain, however few studies have applied it to crowdfunding as well (Bi et al., 2017; Zheng et al., 2016).

In attempting to persuade potential consumers to donate for technology development in reward-based crowdfunding platforms, the value of reward is definitely expected to play a central role. Businesses promise to deliver the technology sometime in future to those patrons who donate a certain amount of currency (less than the expected retail price of the technology when launched in future). So from another perspective, it could be stated that the patrons are basically pre-buying the technology at a certain discount. However, there is always the risk of not getting the technology at all, so the proposed discount should be alluring enough for the consumers to turn into patrons. Given the calculations involved in processing the reward information, decision-making based on reward definitely falls under the central route of ELM. Although not specifically in the technology context, rewards have been found to be a significant motivator for crowdfunding backers (Bretschneider and Leimeister, 2017; Kunz et al., 2017; Weinmann et al., 2018). So we expect the value of reward (or the percentage discount on technology in our context) to be a positive factor in converting a consumer to patron, and hence propose the following hypothesis:

H1: On promising to deliver technology in future as reward for current donations, a high relative difference between expected retail price and requested donation leads to a higher likelihood of receiving donation from potential consumer compared to low relative difference.

When receiving the crowdfunded technology as a reward for donation, another related element of crowdfunding which can be expected to be a significant influencer of donation likelihood is the estimated waiting time of receiving the technology. It is possible that a high waiting time might signal a long development process where many things may go wrong and this may increase the risk perceived by the patron. The consideration of waiting time would require significant cognitive exertion and hence it would also be a central route factor under ELM. While the estimated time of delivery of rewards have been found to have a negative impact on the successful completion of a crowdfunding campaign in general (Kunz et al., 2017), the case of technology projects might turn out to be different as technology development is expected to be a complex process and patron expectations on delivery time may be set accordingly. However, we still expect that low waiting time to receive the technology as reward would be preferred over high waiting time, and hence we propose: 
H2: A high temporal distance between the time of donation and the proposed delivery of technology (as reward) leads to a lower likelihood of receiving donation from potential consumer compared to low temporal distance.

Apart from extrinsic motivation from rewards, it has found that crowdfunding backers are also prosocially motivated and develop feelings for the projects (Bretschneider and Leimeister, 2017). It could be possible that inclusion of affective cues (emotional request, show of gratitude, inspirational quotes on charity, etc.) within donation request context may trigger emotional reactions of altruism and technophilia and lead to higher likelihood of donation. Of course, this would entail heuristic information processing and thus take the peripheral route of ELM. The theme of reward vs. philanthropic motivation (Ryu et al., 2016) along with pro-social behaviour (Bretschneider and Leimeister, 2017) has been touched upon in general crowdfunding as well. With the expectation that affective cues would positively influence potential consumers to donate, we propose the following:

H3: In the donation request context, a high presence of affective cues leads to a higher likelihood of receiving donation from potential consumer compared to low presence of affective cues.

Being closely intertwined, we also expect an interaction effect between the value of reward and the waiting time to receive the reward. In case of low discount on technology as reward, proposing high waiting time should act as further dampener in the motivation to donate. This should lead to a big difference in the donation likelihood between high and low waiting time scenarios in case of low reward. However, when the reward proposed is high, it should provide a cushioning effect and hence we do not expect a huge difference in donation likelihoods of low and high waiting time. Based on this argument, we propose a positive interaction effect between reward and waiting time, and state the following hypothesis:

H4: The difference in the likelihood of receiving donation from potential consumer between low and high temporal distances (to deliver reward) is higher for low reward compared to high reward.

\section{Research Design}

To ensure a thorough testing of the hypotheses, we design a 2 x (2x2) mixed factorial experiment using two levels (Low/High) for each of the three factors: Affective Cues x (Discount x Waiting Time), where first factor is treated between-subjects and rest two are treated within-subjects (4 repeated measures). The details of the experiment is provided in subsections below.

\subsection{Pre-tests}

We started with conducting multiple pre-tests on 13 doctoral students (aged 25 to 46) familiar with latest trends in technology for developing proper treatment conditions for the experiment. Our first aim was to identify some new technology products to be used in the experimental scenarios as product controls, and second aim was to fix low/high levels for the reward/discount and waiting time. A consumers' evaluation of reward/discount could be affected by the price and attractiveness of the technology. Even the evaluation of waiting time could be dependent upon how complex the technology development process would be as per consumer expectation. Hence, in our pre-tests, we gave the respondents a list of 10 trending technologies of different price points (prices not shown to respondents to avoid bias), and asked them to rate them (Likert scale 1-7) based on perceived attractiveness and complexity. In other pre-tests we asked them to imagine pre-ordering a new technology (under development) and asked them to rate their opinions on attractiveness of different discount ranges (in \%ages), price ranges (in US\$) and estimated waiting times for product delivery (in months). Based on the results from all the pre-tests, we judiciously selected 4 technology products ranging from low to high in terms of price, attractiveness, and complexity. And for each technology, we also fixed respective low and high values for discount and waiting time to be used in the experimental scenarios. All these values are provided in Table 1.

\begin{tabular}{lllll}
\hline & $\begin{array}{l}\text { Smart Bluetooth } \\
\text { Speaker }\end{array}$ & $\begin{array}{l}\text { Long Lasting } \\
\text { Smartwatch }\end{array}$ & $\begin{array}{l}\text { Virtual Reality } \\
\text { Headset }\end{array}$ & 3D Printer \\
\hline Perceived Attractiveness (1-7) & 3.27 & 3.47 & 4 & 4.4 \\
Perceived Complexity (1-7) & 2.2 & 2.47 & 3.67 & 4.13 \\
Price (in USD) & $\$ 50$ & $\$ 100$ & $\$ 200$ & $\$ 300$ \\
\hline
\end{tabular}




\begin{tabular}{lllll}
\hline Low Waiting Time (in months) & 1 & 1.5 & 2 & 2.5 \\
High Waiting Time (in months) & 9 & 10 & 11 & 12 \\
Low Patron Discount (in \%age) & 12 & 15 & 18 & 22 \\
High Patron Discount (in \%age) & 50 & 55 & 60 & 65 \\
\hline
\end{tabular}

Table 1. Technology products and treatment levels selected based on pre-tests

\subsection{Experiment Design}

The overall design of our 2x (2x2) mixed factorial experiment is shown is Table 2. Affective cues was kept as a between-subjects factor and not within-subjects since emotional effects tend to stay for longer period and a high-affect treatment in one scenario may distort responses for other low-affect scenarios. So all participants were divided into two major groups, with entire Group A being treated with low level of affective cues, and Group B treated with the high level. Each group was further divided into 4 blocks where participants in each block were presented with 4 different scenarios, each with a different technology and different combination of treatment levels (LL, LH, HL, HH) for discount and waiting time. However, the sequence of these treatments varied cyclically in each block, thus creating a Latin square experimental design as could be seen in Table 2. This is considered to be a much more powerful design compared to just presenting a single sequence of within-subjects treatments. By presenting different sequences to the subjects it systematically eliminates the issue of carry-over effects common with repeated measures designs (Grant, 1948). The design also presents all the treatment combinations to be tested for each technology as well, thus controlling a lot of unknown error-inducing factors related to the interaction of a certain treatment condition with a certain technology.

\begin{tabular}{lllll}
\hline Group A (L Affect) & $\begin{array}{l}\text { Smart Bluetooth } \\
\text { Speaker }\end{array}$ & $\begin{array}{l}\text { Long Lasting } \\
\text { Smartwatch }\end{array}$ & $\begin{array}{l}\text { Virtual Reality } \\
\text { Headset }\end{array}$ & 3D Printer \\
\hline Broup B (H Affect) & LL & LH & HL & HH \\
Block 2 & LH & HL & HH & LL \\
Block 3 & HL & HH & LL & LH \\
Block 4 & HH & LL & LH & HL \\
\hline
\end{tabular}

Note: All scenarios display L/H Waiting Time followed by L/H Discount

Table 2. The $2 x$ (2x2) mixed factorial Latin Square design chosen for our experiment

\subsection{Scenarios and Measures}

For the overall context of the study, the participants were told that their help was required to improve product launch plans and donor rewards proposed by 4 technology startups as presented in the four scenarios. A sample screenshot of an experimental scenario is provided in Appendix 1. In each scenario, the participants were asked to imagine their interest in owning a certain genre of technology and were presented with the proposed plan of a startup to launch a product in that genre sometime in future. Two options were offered to the subjects: A) be a consumer: Wait till product launch and purchase at full retail price; B) be a donor: Provide the requested donation (discounted price of product) right now and the technology will be delivered as reward after launch. The within-subject low/high levels of proposed discount and estimated waiting times were varied in each scenario based on the design discussed earlier. The between-subject factor of affective cues was varied as follows: the low level constituted of the phrase "Kindly donate to support us" and offering a card with "Thank you Patron!" as a mark of gratitude. The high level scenarios had the same abovementioned phrase but also had two additional inspirational quotations (one on altruism and charity, and another on love of technology). Also, instead of a card, the patrons were offered a plaque with "Thank you Patron! You made this possible!"

For measuring the dependent variable of our study, for each scenario we asked the participants to rate (in Likert scale from 1: extremely unlikely to 7: extremely likely) the following: "Based on the given scenario, kindly rate your likelihood of taking the following action: Go with Option B (Donor)". We also used bipolar scales (1: extremely low to 7: extremely high) gauge subjects' opinions on five aspects to be used for manipulation checks and controls: waiting time, discount percentage, attractiveness of 
technology, technology complexity, and price. On completion of the four scenarios, subjects were asked some probing questions to understand their decision-making process, e.g. rate (from 1: strongly disagree to 7: agree) the following statement: "Feeling good about helping startups influenced my likelihood to be a donor". However, given the space and scope restrictions, we won't cover the details and analyses of these questions in this paper. Few more measures for demographic controls were also taken, such as age, gender, and occupation. Further, some psychographic controls were also measures where we asked the subjects to rate themselves on taking risk, helping others, attitude towards technology, donation regularity, and familiarity with technologies.

\subsection{Sample}

Since we essentially intend to study decision-making of technology consumers who may engage in crowdfunding to become patrons, we wanted to ensure that our experimental subjects were not only technology users but also open-minded and favourable towards the idea of donating for development of technologies. Hence, for recruiting our participants, we sent out email invitations to a pool of university students as well as a class of professionals (attending a techno-management course) with a real promise that for every completed response we would donate a certain sum to Wikipedia. Based on the completed responses, our final sample consists of 114 participants with $72 \%$ males and $28 \%$ females. $46.5 \%$ of them are students, $48.25 \%$ are employed professionals, and rest $5.25 \%$ belong to other occupations (selfemployed, unemployed, etc.). The average age of participants is 32 years from a minimum of 22 years to maximum of 50 years. Since each subject were given 4 different treatments, the total number of observations we had to analyse was 456 .

\section{Analyses and Results}

As part of data checks on the 456 observations, we removed those having any missing values. Also, to reduce possibility of spurious data (respondents marking answers without even reading questions), we removed observations where respondents have marked same values for multiple consecutive questions even where it is not possible logically. Next, to test whether the manipulations worked as per intentions, we had included manipulation check questions (on a scale of 1: extremely low to 7: extremely high) related to respondents' perceptions of waiting time and discount. So that in case the perceptions did not match with our intended treatment levels, we could remove the observation. Accordingly, we excluded observations where the treatment level of independent variables is low but their perceived values are greater than 4, and also where the treatment level of independent variables is high but their perceived values are less than 4 . Ultimately, we were left with 327 observations having correctly applied treatments for conducting our analysis.

We used a linear mixed model to analyse the data since it gives us the flexibility not just to estimate the fixed effects of the manipulated and control variables, but also the random effects (found significant) arising from the subjects and repeated effects (also found significant) for using multiple observations from each subject according to the Latin Square design (West et al., 2014). Table 3 summarizes the Type III fixed effects of the independent variables, product controls, demographic controls, psychographic controls, and design controls on the donation likelihood. Significant interaction effect was observed between Waiting Time and Discount $(F(1,230.635)=0.33, p<0.05)$, but all other interaction effects were found to be insignificant. As for the main effects, increasing Waiting Time from low to high had a significant negative impact $(F(1,232.168)=59.53, p<0.05)$ with a decrease in donation likelihood by 1.304 (on a scale of 1 to 7$)$. Increasing Discount from low to high was found to significantly $(F(1,226.454)$ $=131.694, p<0.05$ ) improve the DV by 1.914. The presence or absence of affective cues in the scenarios did not significantly influence the donation likelihoods of the respondents. Thus, all the hypotheses pertaining to the central route of ELM $\left(\mathrm{H}_{1}, \mathrm{H} 2\right.$, and $\left.\mathrm{H}_{4}\right)$ are supported, whereas impact of peripheral route $\left(\mathrm{H}_{3}\right)$ is not supported.

\begin{tabular}{lllll}
\hline DV: Donation Likelihood & $\begin{array}{l}\text { Numerator } \\
\mathrm{df}\end{array}$ & $\begin{array}{l}\text { Denominator } \\
\mathrm{df}\end{array}$ & $\mathrm{F}$ & Sig. \\
\hline $\begin{array}{l}\text { Intercept } \\
\text { Independent Variables: }\end{array}$ & 1 & 153.767 & 12.961 & .000 \\
IV1_WaitingTime & 1 & 232.168 & 59.530 & .000 \\
IV2_Discount & 1 & 226.454 & 131.694 & .000 \\
\hline
\end{tabular}




\begin{tabular}{lllll}
\hline IV3_AffectCues & 1 & 111.452 & .149 & .700 \\
IV1_WaitingTime * IV2_Discount & 1 & 222.664 & 7.291 & .007 \\
IV1_WaitingTime * IV3_AffectCues & 1 & 230.635 & .330 & .566 \\
IV2_Discount * IV3_AffectCues & 1 & 224.714 & .344 & .558 \\
IV1_WaitingTime * IV2_Discount * IV3_AffectCues & 1 & 225.255 & .513 & .475 \\
Product Controls: & & & & \\
Product & 3 & 241.398 & 3.566 & .015 \\
PercAttractiveness & 1 & 314.558 & 16.143 & .000 \\
PercComplexity & 1 & 286.959 & .360 & .549 \\
PercPricing & 1 & 295.891 & 2.824 & .094 \\
Demographic Controls: & & & & \\
Gender & & 100.028 & .570 & .452 \\
Age & 1 & 107.697 & 4.012 & .048 \\
Occupation & 1 & 101.154 & 1.889 & .157 \\
Psychographic Controls: & 2 & & & \\
TakesRisk & & & & \\
HelpsOthers & & & & \\
LikesTech & 1 & 96.615 & 3.346 & .070 \\
DonatesFreq & 1 & 95.375 & 1.034 & .312 \\
TechFamiliarity & 1 & 100.832 & .631 & .429 \\
Design Controls: & 1 & 95.613 & 1.004 & .319 \\
Block & 1 & 103.725 & .431 & .513 \\
\hline
\end{tabular}

Table 3. Fixed effects of factors using linear mixed models

To investigate exactly how the donation likelihoods changed at different levels of waiting time and rewards, we looked at the estimated marginal means (EMM) of the DV on a scale of 1 to 7 using Bonferroni adjustment (Dunn, 1961). In case of low waiting time, the EMM for donation likelihood was found to be 4.211 (Standard Error: 0.243) for low reward scenario, and 5.677 (0.189) for high rewards; whereas in case of high waiting time, the EMM for donation likelihood was found to be 2.459 (0.222) for low reward scenario, and 4.822 (o.225) for high rewards. Fig. 1 plots the points to visually depict the findings. It could be seen that while the donation likelihood rises up as expected on increasing the reward, the increase is higher for high waiting time scenario. This is what caused the interaction effect between waiting time and discount to be significant.

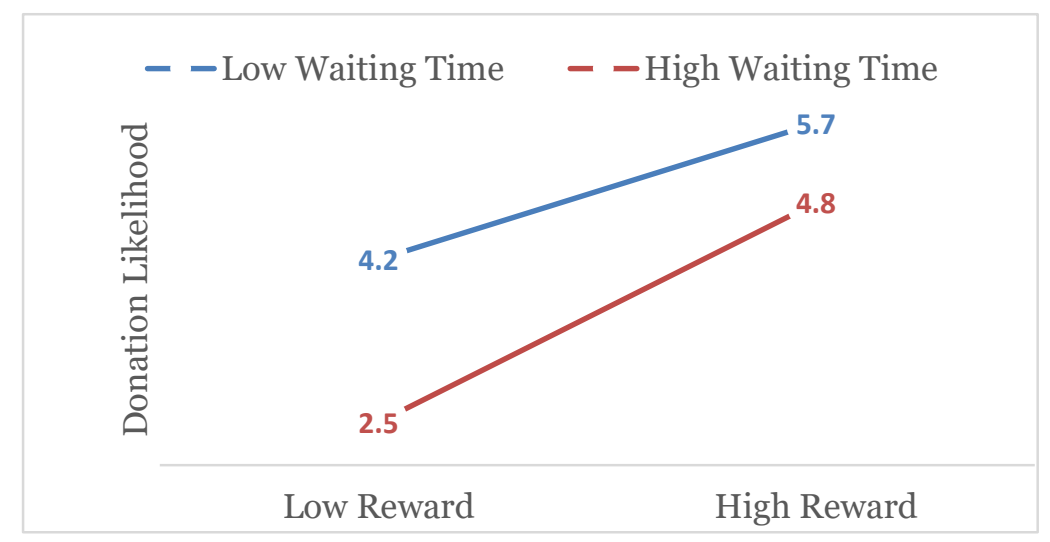

Figure 1: Estimated Marginal Means of Donation Likelihood 


\section{Discussion}

Our research goal was to understand how certain core elements of reward-based crowdfunding impact a business' likelihood of receiving donation (for developing technology) from a potential consumer promised of being rewarded with the technology in future. Our findings show that potential consumers are influenced more by the central extrinsic factors like value of reward and time to receive reward than the peripheral intrinsic factors like altruism and technophilia, when considering whether to indulge in reward-based crowdfunding. This practically implies that technology start-ups should focus more on the deal offered to potential consumers rather than making emotional pitches to affect their decisions. Also, from the significant interaction effect between waiting time and reward we could infer that technology start-ups should be cautious and calculative in designing their crowdfunding offerings as high rewards becomes critical in case of high waiting time scenario without which the crowdfunding campaign would most definitely fail; whereas for low waiting time offering a low to moderate reward is enough to raise donations. Additionally, from looking at the control variables in our model we could gauge that certain technology-related characteristics also play important roles with a significant influence $(p<0.1)$ of the product, its perceived attractiveness, and the expected retail price. So start-ups cannot expect unattractive high-priced products to succeed in crowdfunding campaigns even when offering attractive rewards.

This study makes several theoretical contributions, specifically to the literature on crowdfunding of technologies, and to the IS domain in general. Firstly, this is the first study to investigate the conditions under which technology consumers may prefer to partake in reward-based crowdfunding and thus play the role of technology patrons. There is a dearth of literature on technology patrons, and the findings of this study make a fundamental contribution to this nascent area. Secondly, this study uses a controlled experiment to establish causal links (to the extent practically possible) of certain manipulated factors and their interactions with donation likelihood of potential consumers. Thus, the findings are more focused and reliable compared to association-based studies using secondary data. Thirdly, very few studies have applied the ELM theory to the crowdfunding context (Bi et al., 2017; Zheng et al., 2016), and this study demonstrates yet another application of ELM in reward-based technology crowdfunding context. Our findings showing a clear influence of central route and non-influence of peripheral route provide significant theoretical contributions with potential for further research. Fourthly, rare technology-based constructs like technology attractiveness and technology complexity have been introduced, measured, and used in this paper. These can be further used in technology-centric studies in various contexts. Fifthly, in the IS literature, although experiments are increasingly being used, the more complex designs have rarely been seen. We hope our methodological novelty - mixed designs with Latin Square (Grant, 1948) and using linear mixed models (West et al., 2014) for analysis - would also help other IS researchers understand and consider delving into the richness of experiment designs and analyses.

The findings of this research also have potential tactical implications for technology start-ups planning to raise funding through reward-based crowdfunding and donations. It shows that given the right conditions, a consumer may choose to donate instead of purchase the technology. So the companies can use their sales leads to also find potential patrons. They can tailor their crowdfunding request plans by focusing more on the offerings, like highlighting on how with a small waiting time the consumers can get the same technology at a good discount. Also, they can create interesting product demo videos to enhance technology attractiveness. However, trying to include more affective content in videos or showing how complex the technology development process is, may not bear expected fruitful results (although no negative result as well). Given the interaction between factors, it might be a good idea for companies to pilot test various combinations of their offerings before going to crowdfunding market. For another stakeholder, the crowdfunding platforms, similar implications can be drawn but from a different perspective. They can (re)design their campaign forms and display pages focusing on the significant factors, along with providing guidelines to start-ups on what works.

Finally, we acknowledge certain limitations of this study and the potential areas of future research stemming out of them. Firstly, we could only capture the donation intention and not the actual behaviour of a limited sample. Future studies may try to make the experiment more realistic by actually offering consumers some budget and then propose the options of patronizing or purchasing the product. Secondly, we could only investigate few factors owing to the methodology of our study. However, there are many other factors like reputation of start-ups, peer influences in crowdfunding community, etc. which could be studied in future. Thirdly, we could only provide one reward option for each scenario. However, practically, a technology company can offer a tiered-reward system based on the amount of donation. This complexity can be added in future. Fourthly, we mostly focused on what was happening but not much into the why, which would require delving deeper into the psychology of patrons. 


\section{Conclusion}

We focused on the phenomenon of how reward-based crowdfunding transforms potential consumers into technology patrons by enabling them to donate for technology development in lieu of receiving the finished product as reward in future. Given the dearth of literature specifically on crowdfunding of technologies, not much is known regarding the core factors which convince technology consumers to donate instead of purchase the product. We used the Elaboration Likelihood Model which proposes central and peripheral routes of information processing as our theoretical base, and conducted a 2x2x2 mixed-design experiment to examine the effects of three core elements of crowdfunding (value of reward, waiting time to receive technology, and affective cues in donor appeal) and other control variables (technology attractiveness, complexity, price, etc.) on a potential consumer's likelihood to donate. We found positive impact of reward, and negative impact of waiting time, both of which fall into central route of ELM. There was no observed effect of affective cues which falls into peripheral route. Significant interaction between reward and waiting time was also observed. We presented several theoretical and methodological contributions in IS domain, along with tactical implications for technology start-ups planning to raise funding through donations, and design implications for crowdfunding platforms. We hope this study would encourage other IS researchers to take interest into this nascent but growing field of patronage of technologies.

\section{References}

Banerjee, S. and Bose, I. (2017), "Patronizing Technology: An Emergent Technophilic Behavior”, ICIS 2017 Proceedings.

Beaulieu, T.Y., Sarker, S. and Sarker, S. (2015), "A Conceptual Framework for Understanding Crowdfunding”, Communications of the Association for Information Systems, Vol. 37, pp. 1-31.

Bi, S., Liu, Z. and Usman, K. (2017), "The Influence of Online Information on Investing Decisions of Reward-based Crowdfunding”, Journal of Business Research, Vol. 71, pp. 10-18.

Bretschneider, U. and Leimeister, J.M. (2017), “Not Just an Ego-trip: Exploring Backers' Motivation for Funding in Incentive-based Crowdfunding”, Journal of Strategic Information Systems, Vol. 26 No. 4, pp. 246-260.

Cordova, A., Dolci, J. and Gianfrate, G. (2015), "The Determinants of Crowdfunding Success: Evidence from Technology Projects", Procedia - Social and Behavioral Sciences, Vol. 181, pp. 115-124.

Dunn, O.J. (1961), "Multiple Comparisons among Means", Journal of the American Statistical Association, available at:https://doi.org/10.1080/01621459.1961.10482090.

Grant, D.A. (1948), "The Latin Square Principle in the Design and Analysis of Psychological Experiments", Psychological Bulletin, available at:https://doi.org/10.1037/hoo53912.

Jung, E.J., Susarla, A. and Sambamurthy, V. (2014), "Evolutionary Fundraising Patterns and Entrepreneurial Performance in Crowdfunding Platforms", ICIS-RP, pp. 1-10.

Kickstarter. (2018), “Kickstarter Stats”, available at: https://www.kickstarter.com/help/stats.

Kim, D. and Benbasat, I. (2009), "Trust-Assuring Arguments in B2C E-commerce: Impact of Content, Source, and Price on Trust", Journal of Management Information Systems, Vol. 26 No. 3, pp. 175206.

Kim, T., Por, M.H. and Yang, S.-B. (2017), "Winning the Crowd in Online Fundraising Platforms: The Roles of Founder and Project Features", Electronic Commerce Research and Applications, Vol. 25, pp. 86-94.

Kunz, M.M., Bretschneider, U., Erler, M. and Leimeister, J.M. (2017), “An Empirical Investigation of Signaling in Reward-based Crowdfunding", Electronic Commerce Research, Vol. 17 No. 3, pp. 425-461.

Ma, X., Khansa, L., Deng, Y. and Kim, S.S. (2013), “Impact of Prior Reviews on the Subsequent Review Process in Reputation Systems", Journal of Management Information Systems, available at:https://doi.org/10.2753/MISo742-1222300310. 
Mollick, E. (2014), “The Dynamics of Crowdfunding: An Exploratory Study", Journal of Business Venturing, Vol. 29 No. 1, pp. 1-16.

Petty, R.E. and Cacioppo, J.T. (1983), "Central and Peripheral Routes to Persuasion: Application to Advertising", Advertising and Consumer Psychology, pp. 3-23.

Petty, R.E. and Cacioppo, J.T. (1984), "Source Factors and The Elaboration Likelihood Model of Persuasion", Advances in Consumer Research, available at:https://doi.org/10.1558/ijsll.v14i2.309.

Petty, R.E. and Cacioppo, J.T. (1986), "The Elaboration Likelihood Model of Persuasion", Advances in Experimental Social Psychology, available at:https://doi.org/10.1016/Soo65-2601(08)60214-2.

Ryu, S., Kim, K. and Kim, Y. (2016), "Reward versus Philanthropy Motivation in Crowdfunding Behavior", PACIS 2016 Proceedings.

Weinmann, M., Simons, A., Tietz, M. and Brocke, J.V. (2018), "Get It before It's Gone? How Limited Rewards Influence Backers' Choices in Reward-Based Crowdfunding”, ICIS 2017: Transforming Society with Digital Innovation.

West, B.T., Welch, K.B. and Galecki, A.T. (2014), Linear Mixed Models: A Practical Guide Using Statistical Software, Second Edition, available at:https://doi.org/10.1201/b17198-2.

Zhao, Y., Qin, Y., Zhao, X. and Shi, L. (2018), "Relationship Between Entrepreneurial Motivation and Crowdfunding Success Based on Qualitative Analysis-Based on Kickstarer Website Data”, Wireless Personal Communications, pp. 1-12.

Zheng, H., Hung, J.-L., Qi, Z. and Xu, B. (2016), "The Role of Trust Management in Reward-based Crowdfunding", Online Information Review, Emerald Group Publishing Limited, Vol. 40 No. 1, pp. 97-118.

Zheng, H., Xu, B., Zhang, M. and Wang, T. (2018), "Sponsor's Cocreation and Psychological Ownership in Reward-based Crowdfunding”, Information Systems Journal, available at:https://doi.org/10.1111/isj.12190. 


\section{Appendix 1}

A scenario presented to the respondents of experiment is provided here as an illustration. Each respondent got 4 such scenarios along with several questions to answer.

\section{"Giving is not just about making a donation. It is about making a difference." Kathy Calvin}

"The art challenges the technology, and technology challenges the art." John Lasseter

Imagine that you are interested in owning a long-lasting Virtual Reality Headset. A startup company VR Tech has recently announced their plan to launch a state-of-the-art Virtual Reality Headset as shown below:

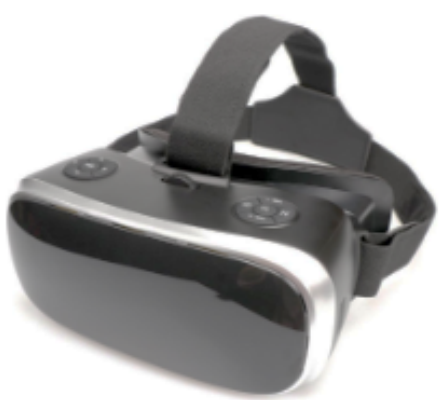

Product prototype (under development). Kindly donate to support us! Top Features:

- Amazing immersive 3D virtual reality experience

- Extremely light weight and comfortable to wear

The technology is still under development and has an estimated launch target of 11 months (subject to the risks/uncertainties inherent in any new technology development process).

The company offers you two options -

\section{Option A: Be our Consumer}

Wait till product launch and then purchase it at full retail price $(\$ 200)$.

\section{Option B: Be our Donor}

Donate $\$ 164 \quad(18 \%$ discount on retail price) right now to support us, and get the product after its launch, along with our heartfelt gratitude (a plaque engraved with 'Thank You Patron! You made this possible!').

\section{Acknowledgements}

We thank Prof. Saravana Jaikumar (Marketing Department, Indian Institute of Management Calcutta) for helping us with his expert counsel on designing the experiment presented in this paper.

\section{Copyright}

Copyright: (C) 2018 Shankhadeep Banerjee and Indranil Bose. This is an open-access article distributed under the terms of the Creative Commons Attribution-NonCommercial 3.0 Australia License, which permits non-commercial use, distribution, and reproduction in any medium, provided the original author and ACIS are credited. 